\title{
TITLE:
}

\section{Linear response theory in the Vlasov equation for homogeneous and for inhomogeneous quasistationary states}

\section{AUTHOR(S):}

Ogawa, Shun; Yamaguchi, Yoshiyuki Y.

\section{CITATION:}

Ogawa, Shun ...[et al]. Linear response theory in the Vlasov equation for homogeneous and for inhomogeneous quasistationary states. Physical Review E 2012, 85(6): 061115.

ISSUE DATE:

2012-06

URL:

http://hdl.handle.net/2433/157926

RIGHT:

(C)2012 American Physical Society. 


\title{
Linear response theory in the Vlasov equation for homogeneous and for inhomogeneous quasistationary states
}

\author{
Shun Ogawa* and Yoshiyuki Y. Yamaguchi \\ Department of Applied Mathematics and Physics, Graduate School of Informatics, Kyoto University, 606-8501 Kyoto, Japan
}

(Received 9 March 2012; published 12 June 2012)

\begin{abstract}
Response to small external forces is investigated in quasistationary states of Hamiltonian systems having long-range interactions. Quasistationary states are recognized as stable stationary solutions to the Vlasov equation, and, hence, the linear response theory to the Vlasov equation is proposed for spatially one-dimensional systems with periodic boundary condition. The proposed theory is applicable both to homogeneous and to inhomogeneous quasistationary states and is demonstrated in the Hamiltonian mean-field model. In the homogeneous case magnetic susceptibility is explicitly obtained, and the Curie-Weiss like law is suggested in a high-energy region. The linear response is also computed in the inhomogeneous case, and resonance absorption is investigated to extract nonequilibrium dynamics in the unforced system. Theoretical predictions are examined by direct numerical simulations of the Vlasov equation.
\end{abstract}

DOI: 10.1103/PhysRevE.85.061115

PACS number(s): 05.20.Dd, 46.40.Ff

\section{INTRODUCTION}

Hamiltonian systems with long-range interactions show macroscopic behavior that differs markedly from ones with short-range interactions, which satisfy assumptions of additivity and extensivity so the equilibrium statistical mechanics and the thermodynamics work $[1,2]$. Such long-range systems are observed as plasma systems, stellar systems, two-dimensional point vortex systems, cold atoms, and mean-field models [1-3]. They are frequently trapped in long-lasting nonequilibrium states called quasistationary states (QSSs), and the lifetime of a QSS is typically read as $N^{\delta}(\delta>0)$, where $N$ is the number of elements. This divergence of lifetime implies that a large system cannot achieve thermal equilibrium within a human's lifetime. Moreover, this divergence is observed in both classical and quantum systems with long-range interactions [4-7]. It is, therefore, interesting to construct a nonequilibrium statistical mechanics and thermodynamics dealing with QSSs.

A useful tool to investigate QSSs is the Vlasov equation by which temporal evolution of a Hamiltonian system with longrange interactions is described in the limit $N \rightarrow \infty$ [8-10]. We remark that the Vlasov equation is also called the collisionless Boltzmann equation [2]. Using an incompressive feature of the Vlasov equation, a nonequilibrium statistical mechanics is proposed for self-gravitating systems [11]. The theory is not fully successful in the self-gravitating systems [12-14] but gives a good prediction of a QSS for a given waterbag initial state in the Hamiltonian mean-field (HMF) model [15-18].

The statistical mechanics for QSSs has been considered in a system without external fields. To construct thermodynamics in QSSs, it is important to understand response to external fields or external forces which represent operations, for instance, adiabatic compression for gas confined in a box, and exerting external magnetic field for a magnetic material. We stress that we must describe such operations and theory of

\footnotetext{
*sogawa@amp.i.kyoto-u.ac.jp
}

response to the external fields in the context of the Vlasov dynamics, since QSSs are recognized as stable stationary solutions to the Vlasov equation [4]. Another important topic in investigating the linear response is that one can obtain nonequilibrium dynamics in unforced systems by exerting weak probe fields and measuring linear response to these fields.

Writing the linear response theory based on the Vlasov equation has an advantage against the Kubo formula based on the Liouville equation. The Kubo formula requires solving equations of motion for an $\mathrm{N}$-body Hamiltonian system [19,20], but an $N$-body system is nonintegrable in general. On the other hand, the Vlasov equation is described by a single-body Hamiltonian, and the system is integrable when spatial dimension is one and a considering state is stationary.

The linear response theory in the Vlasov equation is also proposed by Patelli et al. [21]. We clarify advantages of the present paper against the previous work: (a) They assume that conjugate variables of external field depend on position variables only, but we do not require this assumption in a general theory. (b) They do not deal with response around spatially inhomogeneous QSSs, but the present theory includes both homogeneous and inhomogeneous cases. (c) The present paper investigates the Curie-Weiss law like behavior of zero-field susceptibility for some QSSs. (d) We use the linear response theory to extract nonequilibrium dynamics in inhomogeneous states of the unforced HMF model. We try to probe the Landau poles by resonance absorption instead of directly solving the dispersion relation.

This article is constructed as follows. We propose the explicit but formal linear response theory based on the Vlasov equation for spatially periodic one-dimensional systems in Sec. II. The linear response is described by two families of functions, and concrete forms of the functions are given in Sec. III both for homogeneous and for inhomogeneous cases. The theory is applied for the HMF model in Sec. IV and is numerically examined for the homogeneous case in Sec. V and for the inhomogeneous case in Sec. VI. Section VII is devoted to a summary and discussions. 


\section{LINEAR RESPONSE THEORY}

\section{A. Model}

We consider a spatially periodic one-dimensional Hamiltonian system consisting of $N$ particles and add a small external field to the system. Let the Hamiltonian of this system be

$$
\begin{aligned}
H_{N} & \left(q_{1}, \ldots, q_{N}, p_{1}, \ldots, p_{N}, t\right) \\
= & \sum_{i=1}^{N} \frac{p_{i}^{2}}{2}+\frac{1}{N} \sum_{i<j} V\left(q_{i}-q_{j}\right)+\sum_{i=1}^{N} H_{1}\left(q_{i}, p_{i}, t\right) .
\end{aligned}
$$

The position $q_{i}$ is defined on a circle and its domain is $q_{i} \in$ $[-\pi, \pi)$, and $p_{i} \in \mathbb{R}$ is the conjugate momentum. The terms of $H_{1}$ represent energy added by the time-dependent external field. In the large $N$ limit, temporal evolution of the system is described by the single-body distribution function $f$ obeying the Vlasov equation,

$$
\frac{\partial f}{\partial t}+\{\mathcal{H}[f], f\}=0 .
$$

The single-body Hamiltonian is expressed by [21]

$$
\mathcal{H}[f](q, p, t)=\mathcal{H}_{0}[f](q, p, t)+H_{1}(q, p, t),
$$

where

$$
\begin{gathered}
\mathcal{H}_{0}[f](q, p, t)=\frac{p^{2}}{2}+\mathcal{V}[f](q, t), \\
\mathcal{V}[f](q, t)=\iint V\left(q-q^{\prime}\right) f\left(q^{\prime}, p, t\right) d q^{\prime} d p,
\end{gathered}
$$

and $\{f, g\}$ is the Poisson bracket defined by

$$
\{f, g\}=\frac{\partial f}{\partial p} \frac{\partial g}{\partial q}-\frac{\partial f}{\partial q} \frac{\partial g}{\partial p} .
$$

\section{B. Implicit linear response}

For investigating linear response around a stationary state, we set $H_{1}=0$ for $t<0$ and add the small external field at $t=0$. Before adding the external field, the Vlasov equation is written as

$$
\frac{\partial f}{\partial t}+\left\{\mathcal{H}_{0}[f], f\right\}=0 .
$$

Let $f_{0}(q, p)$ be a stationary solution to the Vlasov equation with $H_{1}=0$ and, hence, satisfy

$$
\left\{\mathcal{H}_{0}\left[f_{0}\right], f_{0}\right\}=0
$$

at any time $t$. The added small external field represented by $H_{1}$ induces a small change of the distribution function from $f_{0}$, and we write the modified distribution function as

$$
f(q, p, t)=f_{0}(q, p)+f_{1}(q, p, t) .
$$

We assume that amplitude of $f_{1}$ is the same order with or smaller than $H_{1}$. Substituting (9) into the Vlasov equation (2), we obtain the linearized Vlasov equation

$$
\frac{\partial f_{1}}{\partial t}=-\left\{\mathcal{H}_{0}\left[f_{0}\right], f_{1}\right\}-\left\{\mathcal{V}\left[f_{1}\right]+H_{1}, f_{0}\right\}
$$

where the higher-order term $\left\{\mathcal{V}\left[f_{1}\right]+H_{1}, f_{1}\right\}$ was omitted. Introducing the operator $\mathcal{L}_{0}$ defined by

$$
\mathcal{L}_{0} f_{1}=-\left\{\mathcal{H}_{0}\left[f_{0}\right], f_{1}\right\},
$$

the linearized Vlasov equation is rewritten by

$$
\frac{\partial f_{1}}{\partial t}=\mathcal{L}_{0} f_{1}-\left\{\mathcal{V}\left[f_{1}\right]+H_{1}, f_{0}\right\} .
$$

The formal solution to (12) is, noting that $f_{1}=0$ for $t<0$,

$$
f_{1}(q, p, t)=-\int_{0}^{t} e^{(t-s) \mathcal{L}_{0}}\left\{\mathcal{V}\left[f_{1}\right](s)+H_{1}(s), f_{0}\right\} d s,
$$

where we omitted arguments in the right-hand-side except for the time.

Let us introduce an observable $B$ which is a smooth function on $\mu$ space, the $(q, p)$ plane. Response to the small external field is observed by the expected value of $B$ with respect to $f_{1}$ defined by

$$
\langle B\rangle_{1}(t)=\iint_{\mu} B(q, p) f_{1}(q, p, t) d q d p .
$$

Substituting the formal solution (13) into (14), we have

$$
\langle B\rangle_{1}(t)=-\int_{0}^{t} d s \iint_{\mu} d q d p B_{t-s}(q, p)\left\{\mathcal{V}\left[f_{1}\right](s)+H_{1}(s), f_{0}\right\} .
$$

The new function $B_{t}(q, p)$ is defined by

$$
B_{t}(q, p)=\left(B \circ \phi_{0}^{t}\right)(q, p),
$$

where $\phi_{0}^{t}$ is the Hamiltonian flow induced by the Hamiltonian $\mathcal{H}_{0}\left[f_{0}\right]$. See Appendix A for deriving (15).

The expression (15) is the linear response of $B$ for the small external field but is implicit since the unknown function $f_{1}$ appears both in the left- and right-hand sides. We will write $\mathcal{V}\left[f_{1}\right]$ by known quantities and give an explicit linear response in the next section.

\section{Explicit linear response}

Thanks to the periodic boundary condition, any functions of $q$ are periodic and, hence, can be expanded in the Fourier series as

$$
f_{1}(q, p, t)=\sum_{k \in \mathbb{Z}} f_{1, k}(p, t) e^{i k q}
$$

and

$$
V(q)=\sum_{k \in \mathbb{Z}} V_{k} e^{i k q} .
$$

Substituting (17) and (18) into (5), we have

$$
\mathcal{V}\left[f_{1}\right](q, t)=2 \pi \sum_{k \in \mathbb{Z}} V_{k} e^{i k q} \rho_{1, k}(t),
$$

where

$$
\rho_{1, k}(t)=\frac{1}{2 \pi} \iint e^{-i k q} f_{1}(q, p, t) d q d p=\frac{1}{2 \pi}\left\langle e^{-i k q}\right\rangle_{1}
$$

is the Fourier transform of the density function $\rho_{1}$ defined by

$$
\rho_{1}(q, t)=\int f_{1}(q, p, t) d p
$$

We substitute (19) into (15) and choose $B$ as $e^{-i k q}$ to obtain an equation for $\rho_{1, k}(t)$. For later convenience, we write the 
exponential factor as a function on $\mu$ space as

$$
E^{k}(q, p)=e^{-i k q},
$$

where $E^{k}$ has the argument $p$ but does not depend on $p$. We then obtain

$$
\begin{aligned}
2 \pi \rho_{1, k}(t)= & -\iint_{\mu} d q d p \int_{0}^{t} d s E_{t-s}^{k} \\
& \times\left[2 \pi \sum_{l \in \mathbb{Z}} V_{l} E^{-l} \rho_{1, l}(s)+H_{1}(s), f_{0}\right] .
\end{aligned}
$$

To treat the convolution with respect to time in Eq. (23), we perform the Laplace transform, which is defined by

$$
\hat{\varphi}(\omega)=\int_{0}^{\infty} \varphi(t) e^{i \omega t} d t, \quad(\operatorname{Im} \omega>0)
$$

for a function $\varphi(t)$. The condition $\operatorname{Im} \omega>0$ ensures convergence of the Laplace transform for nondiverging $\varphi(t)$. The equation for $\rho_{1, k}$, Eq. (23), is then transformed to

$$
\widehat{\rho}_{1, k}(\omega)=\sum_{l \in \mathbb{Z}} F_{k l}(\omega) \widehat{\rho}_{1, l}(\omega)+G_{k}(\omega)
$$

where

$$
F_{k l}(\omega)=-V_{l} \iint \widehat{E_{\omega}^{k}}(q, p)\left\{E^{-l}, f_{0}\right\} d q d p
$$

and

$$
G_{k}(\omega)=-\frac{1}{2 \pi} \iint \widehat{E_{\omega}^{k}}(q, p)\left\{\widehat{H}_{1}(\omega), f_{0}\right\} d q d p .
$$

Let us introduce the vectors $\widehat{\rho}_{1}(\omega)=\left(\widehat{\rho}_{1, k}(\omega)\right), \quad G(\omega)=$ $\left(G_{k}(\omega)\right)$ and the matrix $F(\omega)=\left(F_{k l}(\omega)\right)$. Equation (25) is, thus, formally written in a simple form as

$$
\widehat{\rho}_{1}(\omega)=F(\omega) \widehat{\rho}_{1}(\omega)+G(\omega),
$$

and the formal solution is

$$
\widehat{\rho}_{1}(\omega)=(I-F(\omega))^{-1} G(\omega),
$$

where $I$ is the identity matrix.

Let us come back to rewrite the implicit linear response (15) into an explicit linear response. The Laplace transform of $\mathcal{V}\left[f_{1}\right]$ is, from (19),

$$
\begin{aligned}
\widehat{\mathcal{V}\left[f_{1}\right]}(q, \omega) & =2 \pi \sum_{k \in \mathbb{Z}} V_{k} e^{i k q} \widehat{\rho}_{1, k}(\omega)=v \cdot \widehat{\rho}_{1}(\omega) \\
& =v(q) \cdot(I-F(\omega))^{-1} G(\omega)
\end{aligned}
$$

where the vector $v(q)$ is defined by $v(q)=\left(2 \pi V_{k} e^{i k q}\right)$ and $v \cdot \widehat{\rho}_{1}$ represents the Euclidean inner product between $v$ and $\widehat{\rho}_{1}$. Finally, the Laplace transformed linear response is expressed in the form

$$
\begin{aligned}
{\widehat{\langle B\rangle_{1}}}_{1}(\omega)= & -\iint \widehat{B_{\omega}}\left\{v \cdot(I-F(\omega))^{-1} G(\omega)\right. \\
& \left.+\widehat{H_{1}}(\omega), f_{0}\right\} d q d p .
\end{aligned}
$$

We give some remarks on (31). (i) The right-hand side of (31) does not include $f_{1}$ and consists of the given external field $H_{1}$ and quantities computed from $f_{0}$. (ii) The external field $H_{1}$ and the observable $B$ are functions of both $q$ and $p$, while the previous work assumed that they are functions of $q$ only [21]. (iii) The stationary solution $f_{0}$ is not assumed as spatially homogeneous. (iv) An important step to compute the right-hand side of (31) is to obtain $\widehat{E_{\omega}^{k}}$, which gives $F$ and $G$ functions. (v) The expression (31) is explicit but formal in general, since the size of matrix $F$ may be infinite.

We also remark that the relation

$$
\iint \phi\left\{\psi, f_{0}\right\} d q d p=\iint\{\phi, \psi\} f_{0} d q d p=\langle\{\phi, \psi\}\rangle_{0}
$$

can be shown for smooth functions $\phi$ and $\psi$ when $f_{0}$ is a rapidly decreasing function of $p$ in the large $|p|$ by using integration by parts and the periodic boundary condition for $q$. Here $\langle B\rangle_{0}$ is the expected value of $B$ with respect to $f_{0}$. Thanks to the relation (32), the linear response (31), $F$ function (26) and $G$ function (27) are rewritten in the forms of

$$
\begin{gathered}
\widehat{\langle B\rangle_{1}}(\omega)=-\left\langle\left\{\widehat{B_{\omega}}, v \cdot(I-F(\omega))^{-1} G(\omega)+\widehat{H_{1}}(\omega)\right\}\right\rangle_{0}, \\
F_{k l}(\omega)=-V_{l}\left\langle\left\{\widehat{E_{\omega}^{k}}, E^{-l}\right\}\right\rangle_{0}
\end{gathered}
$$

and

$$
G_{k}(\omega)=-\frac{1}{2 \pi}\left\langle\left\{\widehat{E}_{\omega}^{k}, \widehat{H}_{1}(\omega)\right\}\right\rangle_{0},
$$

respectively.

\section{III. $F$ AND $G$ FUNCTIONS}

The linear response (31) is determined by the $F$ and $G$ functions. We therefore explicitly compute $F$ and $G$ functions both for spatially homogeneous and inhomogeneous stationary state $f_{0}$. We assume that $f_{0}$ depends on $(q, p)$ only through $\mathcal{H}_{0}\left[f_{0}\right]$, and then $f_{0}(q, p)=\tilde{f}_{0}\left(\mathcal{H}_{0}\left[f_{0}\right](q, p)\right)$ is a stationary solution satisfying (8).

\section{A. Homogeneous case}

Let us consider the case that the stationary state $f_{0}$ is spatially homogeneous. Omitting the constant of potential, which is irrelevant to dynamics, the single-body Hamiltonian $\mathcal{H}_{0}\left[f_{0}\right]$ is written as

$$
\mathcal{H}_{0}\left[f_{0}\right]=\frac{p^{2}}{2}
$$

The Hamiltonian flow $\phi_{0}^{t}$ is, hence, expressed by the simple form of

$$
\phi_{0}^{t}(q, p)=(q+t p, p) .
$$

The function $E_{t}^{k}(q, p)$ is, therefore,

$$
E_{t}^{k}(q, p)=e^{-i k(q+t p)},
$$

and its Laplace transform is

$$
\widehat{E_{\omega}^{k}}(q, p)=\frac{-e^{-i k q}}{i(\omega-k p)} .
$$

Performing the Fourier series expansion of $H_{1}$ with respect to $q$, the $F$ function and the $G$ function are expressed by

$$
F_{k l}(\omega)=-2 \pi k V_{k} \delta_{k, l} \int_{L} \frac{f_{0}^{\prime}(p)}{\omega-k p} d p
$$


and

$$
G_{k}(\omega)=-k \int_{L} \frac{f_{0}^{\prime}(p)}{\omega-k p} \widehat{H}_{1, k}(p, \omega) d p .
$$

The functions (40) and (41) vanish for $k=0$ and we may set $k \neq 0$. The functions are defined in the upper half $\omega$ plane due to the Laplace transform (24), and we must consider analytic continuation for extending the domain to the whole $\omega$ plane. The integral path $L$ is the real $p$ axis for $\operatorname{Im} \omega>0$, but is continuously modified to avoid the singularity at $p=\omega / k$ for $\operatorname{Im} \omega \leqslant 0$ as $\operatorname{Im} \omega$ goes down. The functions, hence, pick up contribution from the pole for $\operatorname{Im} \omega \leqslant 0$ in addition to the integral on the real $p$ axis $[22,23]$.

The matrix $F$ is diagonal, and the equation (28) can be solved in each element as

$$
\widehat{\rho}_{1, k}(\omega)=\frac{G_{k}(\omega)}{D_{k}(\omega)},
$$

where

$$
D_{k}(\omega)=1+2 \pi k V_{k} \int_{L} \frac{f_{0}^{\prime}(p)}{\omega-k p} d p
$$

is the dielectric function or the dispersion function. Moreover, if $H_{1}$ does not depend on $p$, then we can write the density $\widehat{\rho}_{1, k}$ by

$$
\widehat{\rho}_{1, k}(\omega)=\frac{\widehat{H}_{1, k}(\omega)}{2 \pi V_{k}} \frac{1-D_{k}(\omega)}{D_{k}(\omega)}
$$

for modes of $V_{k} \neq 0$. We note that $V_{k}$ is defined in (18), and it is not a Fourier coefficient of $\mathcal{V}\left[f_{0}\right]$ but of $V(q)$. There must be some nonzero coefficients $V_{k}$ for $k \neq 0$ even for homogeneous $f_{0}$, since the Hamiltonian system (1) is supposed to have longrange interactions.

We exhibit trivial but important examples of the linear response. We assume that (32) holds. Choosing the observable $B(q, p)$ in (15) as a function $g(p)$ depending on $p$ only, $g_{t}$ is identical with $g$ by (37). The linear response of $g$ is, hence,

$$
\langle g\rangle_{1}=\int_{0}^{t} d s \iint d q d p\left\{g_{t-s}, f_{0}\right\}\left[\mathcal{V}\left[f_{1}\right](s)+H_{1}(s)\right]=0,
$$

since $\left\{g_{t-s}, f_{0}\right\}=0$. Setting $g(p)=p^{2}$, we obtain that the kinetic temperature $T_{\text {kin }}=\left\langle p^{2}\right\rangle$ is not affected by the external field within accuracy of order $O\left(H_{1}\right)$. We further remark that the vanishing expectation value (45) holds in systems with periodic boundary condition but may break in a system, for instance, gas confined by walls, since the relation (32) is not guaranteed to hold.

\section{B. Inhomogeneous case}

Suppose that the stationary state $f_{0}$ is spatially inhomogeneous. To get the Hamiltonian flow $\phi_{0}^{t}$, we transform the Cartesian coordinate $(q, p)$ to the angle-action variables $(\theta, J)$. This transform is not always injective on the whole $\mu$ space and must be defined on each of divided spaces to make the transform bijective. We skip details of this division. See Ref. [24] for the HMF case.
The single-body Hamiltonian is integrable and is a function of $J$ only as $\mathcal{H}_{0}\left[f_{0}\right](J)$. Defining frequency

$$
\Omega(J)=\frac{d \mathcal{H}_{0}[f]}{d J}(J),
$$

the Hamiltonian flow $\phi_{0}^{t}$ is written as

$$
\phi_{0}^{t}(\theta, J)=(\theta+t \Omega(J), J) \text {. }
$$

Let us compute $\widehat{E_{\omega}^{k}}$, which appears in $F$ and $G$ functions, by using the angle-action variables. The function $E^{k}$ is periodic with respect to $\theta$, and, hence, it is expanded in the Fourier series as

$$
E^{k}(\theta, J)=e^{-i k q(\theta, J)}=\sum_{m \in \mathbb{Z}} E^{k, m}(J) e^{i m \theta},
$$

where

$$
E^{k, m}(J)=\frac{1}{2 \pi} \int_{-\pi}^{\pi} e^{-i k q(\theta, J)} e^{-i m \theta} d \theta .
$$

Using (47), the function $E_{t}^{k}$ is expressed by

$$
E_{t}^{k}(\theta, J)=\sum_{m \in \mathbb{Z}} E^{k, m}(J) e^{i m(\theta+t \Omega(J))},
$$

and the function $\widehat{E_{\omega}^{k}}$ is

$$
\widehat{E_{\omega}^{k}}(\theta, J)=\sum_{m \in \mathbb{Z}} E^{k, m}(J) e^{i m \theta} \frac{-1}{i[\omega+m \Omega(J)]} .
$$

The transform $(q, p) \mapsto(\theta, J)$ is canonical, and, hence, the Poisson bracket is invariant under this transform, and $d q \wedge$ $d p=d \theta \wedge d J$ holds. The $F$ and $G$ functions are, thus,

$$
F_{k l}(\omega)=-2 \pi V_{l} \sum_{m \in \mathbb{Z}} m \int_{L} \frac{f_{0}^{\prime}(J)}{\omega-m \Omega(J)} E^{k,-m}(J) E^{-l, m}(J) d J
$$

and

$$
G_{k}(\omega)=-\sum_{m \in \mathbb{Z}} m \int_{L} \frac{f_{0}^{\prime}(J)}{\omega-m \Omega(J)} E^{k,-m}(J) \widehat{H}_{1, m}(J, \omega) d J,
$$

respectively. The functions (52) and (53) are defined in the upper half $\omega$ plane, and the integral path $L$ lies on the real axis. As done in the homogeneous case, the path $L$ is modified to obtain analytic continuation to the whole $\omega$ plane.

We note that the vanishing expectation value (45) is not always guaranteed for inhomogeneous stationary state even if we set $g$ as a function of $J$ only. The domain of $J$ is, for instance, $[0, \infty)$, and contribution from $J=0$ may remain in the relation (32) in the way of integration by parts.

\section{LINEAR RESPONSE THEORY IN HAMILTONIAN MEAN-FIELD MODEL}

We apply the linear response theory to the HMF model. We summarize the theory for HMF model in this section and examine the theory in the following two sections, Secs. V and VI, for homogeneous and for inhomogeneous cases, respectively. 
The HMF model is a ferromagnetic model and is expressed by taking

$$
V(q)=-\cos q .
$$

We introduce magnetization $\left(M_{x}, M_{y}\right)$ as

$$
\begin{aligned}
& M_{x}[f](t)=\iint f(q, p, t) \cos q d q d p \\
& M_{y}[f](t)=\iint f(q, p, t) \sin q d q d p
\end{aligned}
$$

and denote the response parts by $M_{1, x}(t)=M_{x}\left[f_{1}\right](t)$ and $M_{1, y}(t)=M_{y}\left[f_{1}\right](t)$. The single-body Hamiltonian for the HMF model is

$$
\mathcal{H}_{0}[f](q, p, t)=\frac{p^{2}}{2}-M_{x}[f](t) \cos q-M_{y}[f](t) \sin q,
$$

and the external field $H_{1}$ can be set by

$$
H_{1}(q, p, t)=-h_{x}(t) \cos q-h_{y}(t) \sin q,
$$

where $h_{x}$ and $h_{y}$ are time-dependent external magnetic fields for the $x$ direction and the $y$ direction, respectively.

Using $\cos q$ and $\sin q$ instead of $e^{i q}$ and $e^{-i q}$, and writing them as

$$
C(q, p)=\cos q, \quad S(q, p)=\sin q,
$$

we obtain

$$
\left(\begin{array}{l}
\widehat{M}_{1, x}(\omega) \\
\widehat{M}_{1, y}(\omega)
\end{array}\right)=\left(\begin{array}{ll}
F_{c c}(\omega) & F_{c s}(\omega) \\
F_{s c}(\omega) & F_{s s}(\omega)
\end{array}\right)\left(\begin{array}{l}
\widehat{M}_{1, x}(\omega) \\
\widehat{M}_{1, y}(\omega)
\end{array}\right)+\left(\begin{array}{l}
G_{c}(\omega) \\
G_{s}(\omega)
\end{array}\right),
$$

where

$$
\begin{aligned}
F_{c c}(\omega) & =\iint \widehat{C}_{\omega}\left\{C, f_{0}\right\} d q d p, \\
F_{c s}(\omega) & =\iint \widehat{C}_{\omega}\left\{S, f_{0}\right\} d q d p, \\
F_{s c}(\omega) & =\iint \widehat{S}_{\omega}\left\{C, f_{0}\right\} d q d p, \\
F_{s s}(\omega) & =\iint \widehat{S}_{\omega}\left\{S, f_{0}\right\} d q d p,
\end{aligned}
$$

and

$$
\begin{aligned}
G_{c}(\omega) & =-\iint \widehat{C}_{\omega}\left\{\widehat{H}_{1}(\omega), f_{0}\right\} d q d p, \\
G_{s}(\omega) & =-\iint \widehat{S}_{\omega}\left\{\widehat{H}_{1}(\omega), f_{0}\right\} d q d p .
\end{aligned}
$$

The external field (57) gives

$$
\left(\begin{array}{l}
G_{c}(\omega) \\
G_{s}(\omega)
\end{array}\right)=\left(\begin{array}{ll}
F_{c c}(\omega) & F_{c s}(\omega) \\
F_{s c}(\omega) & F_{s s}(\omega)
\end{array}\right)\left(\begin{array}{l}
\widehat{h}_{x}(\omega) \\
\widehat{h}_{y}(\omega)
\end{array}\right),
$$

and, hence, (59) is solved by

$$
\left(\begin{array}{c}
\widehat{M}_{1, x}(\omega) \\
\widehat{M}_{1, y}(\omega)
\end{array}\right)=(I-F(\omega))^{-1} F(\omega)\left(\begin{array}{l}
\widehat{h}_{x}(\omega) \\
\widehat{h}_{y}(\omega)
\end{array}\right) .
$$

The off diagonal elements of the matrix $F$ vanish as

$$
F_{c s}(\omega)=F_{s c}(\omega)=0
$$

in the homogeneous case. The relation (64) is also satisfied in the inhomogeneous case [24]. The response of magnetization is therefore written as

$$
\begin{aligned}
\widehat{M}_{1, x}(\omega) & =\frac{1-D_{x}(\omega)}{D_{x}(\omega)} \widehat{h}_{x}(\omega), \\
\widehat{M}_{1, y}(\omega) & =\frac{1-D_{y}(\omega)}{D_{y}(\omega)} \widehat{h}_{y}(\omega),
\end{aligned}
$$

where

$$
D_{x}(\omega)=1-F_{c c}(\omega), \quad D_{y}(\omega)=1-F_{s s}(\omega)
$$

are the dispersion functions for the $x$ direction and the $y$ direction, respectively. The concrete forms of $F_{c c}(\omega)$ and $F_{s s}(\omega)$ are expressed by

$$
F_{c c}(\omega)=F_{s s}(\omega)=-\pi \int_{L} \frac{f_{0}^{\prime}(p)}{p-\omega} d p
$$

for the homogeneous case and

$$
\begin{aligned}
& F_{c c}(\omega)=-2 \pi \sum_{m} \int_{L} \frac{m f_{0}^{\prime}(J)}{m \Omega(J)-\omega}\left|C^{m}(J)\right|^{2} d J, \\
& F_{s s}(\omega)=-2 \pi \sum_{m} \int_{L} \frac{m f_{0}^{\prime}(J)}{m \Omega(J)-\omega}\left|S^{m}(J)\right|^{2} d J,
\end{aligned}
$$

for the inhomogeneous case. Here we introduced new functions,

$$
\begin{aligned}
C^{m}(J) & =\frac{1}{2 \pi} \int \cos q(\theta, J) e^{-i m \theta} d \theta, \\
S^{m}(J) & =\frac{1}{2 \pi} \int \sin q(\theta, J) e^{-i m \theta} d \theta .
\end{aligned}
$$

We note that the expression (65) is valid both for homogeneous and for inhomogeneous cases by selecting $F_{c c}$ and $F_{s s}$ as (67) for the homogeneous case and as (68) for the inhomogeneous case.

Let us consider the asymptotic behavior of $M_{1, x}$ and $M_{1, y}$. There is no difference between $M_{1, x}$ and $M_{1, y}$ in (65) formally, and, hence, we focus on $M_{1, x}$ by setting

$$
h_{x}(t)=h \Theta(t) \cos \omega_{0} t,
$$

where $\Theta(t)$ is the step function. The Laplace transform of $h_{x}(t)$ is

$$
\widehat{h}_{x}(\omega)=\frac{-h}{2 i}\left(\frac{1}{\omega-\omega_{0}}+\frac{1}{\omega+\omega_{0}}\right)
$$

and, hence, temporal evolution $M_{1, x}(t)$ is

$M_{1, x}(t)=\frac{-h}{4 \pi i} \int_{\Gamma} \frac{1-D_{x}(\omega)}{D_{x}(\omega)}\left(\frac{1}{\omega-\omega_{0}}+\frac{1}{\omega+\omega_{0}}\right) e^{-i \omega t} d \omega$,

where $\Gamma$ is a Bromwich contour running from $-\infty+i \sigma$ to $+\infty+i \sigma$ with a real positive $\sigma$, which is set such that all singularities of the integrand are below of this Bromwich contour. In inhomogeneous case $D_{x}(\omega)$ has logarithmic singularities and branch points are on the real axis [24,25]. However, we neglect the logarithmic singularities and concentrate on pole singularities. In other words we assume that $\omega_{0}$ is not at the branch points. We will discuss on the logarithmic singularities in Sec. VII. 
Suppose that all roots of $D_{x}(\omega)$ are on the lower half $\omega$ plane, and they give exponential Landau dampings [22,23]. Asymptotic behavior of $M_{1, x}(t)$ is then determined by the poles at $\omega= \pm \omega_{0}$ and

$$
M_{1, x}(t) \rightarrow \frac{h}{2}\left[\frac{1-D_{x}\left(\omega_{0}\right)}{D_{x}\left(\omega_{0}\right)} e^{-i \omega_{0} t}+\frac{1-D_{x}\left(-\omega_{0}\right)}{D_{x}\left(-\omega_{0}\right)} e^{i \omega_{0} t}\right] .
$$

In particular, setting $\omega_{0}=0$, we have

$$
M_{1, x}(t) \rightarrow h \frac{1-D_{x}(0)}{D_{x}(0)} .
$$

For the external field of the $y$ direction,

$$
h_{y}(t)=h \Theta(t) \cos \omega_{0} t,
$$

we obtain the same results for $M_{1, y}(t)$ by replacing $x$ with $y$ in Eqs. (73) and (74).

We remark on asymptotic values of $M_{1, x}(t)$ and $M_{1, y}(t)$ for $\omega_{0}=0$. Let us focus on $M_{1, x}(t)$ without loss of generality. We used the step function $\Theta(t)$ in $h_{x}(t)$, and the Laplace transform of $h_{x}(t)$ is therefore expressed by

$$
\widehat{h}_{x}(\omega)=\frac{-h}{i \omega}
$$

for $\omega_{0}=0$. This singularity at the origin $\omega=0$ leads the asymptotic form (74) by the inverse Laplace transform. However, the step function is not essential to give the singularity at the origin $\omega=0$, and any smooth functions give the same singularity if the functions go to a constant in the limit $t \rightarrow \infty$. See Appendix B for details.

\section{RESPONSE IN HOMOGENEOUS CASE}

We add a nonoscillating external field, say, $\omega_{0}=0$, and use (74) to investigate Curie-Weiss-law-like behavior of the susceptibility in homogeneous case. We may set the external field points to the $x$ direction without loss of generality thanks to rotational symmetry in the homogeneous case. The external field is, hence, set as $\left(h_{x}(t), h_{y}(t)\right)=(h \Theta(t), 0)$. We will use energy $U$ to identify the critical point, where energy in the HMF model is defined by

$$
U=\iint \frac{p^{2}}{2} f d q d p+\frac{1}{2}-\frac{M_{x}^{2}+M_{y}^{2}}{2} .
$$

The constant $1 / 2$ in the right-hand side is added for convenience of comparison with previous studies of the HMF model.

\section{A. Thermal equilibrium}

The first example is thermal equilibrium,

$$
f_{0}(p)=\frac{1}{2 \pi} \sqrt{\frac{\beta}{2 \pi}} e^{-\beta p^{2} / 2} .
$$

The critical energy of a second-order phase transition is $U_{\mathrm{c}}=$ $3 / 4$, which corresponds to the critical temperature $T_{\mathrm{c}}=1 / 2$. We chose the unit so the Boltzmann's constant is unity, $k_{B}=1$.

The dispersion function at $\omega=0$ is

$$
D(0)=1-\frac{\beta}{2}
$$

and the asymptotic linear response of $M_{1, x}$ is

$$
M_{1, x}=h \frac{\beta / 2}{1-\beta / 2}=h \frac{T_{\mathrm{c}}}{T-T_{\mathrm{c}}} .
$$

The susceptibility $\chi$, defined by

$$
\chi=\lim _{h \rightarrow 0} \frac{M_{1, x}}{h}=\frac{T_{\mathrm{c}}}{T-T_{\mathrm{c}}},
$$

and $\chi$ diverges at the critical point. This susceptibility is rewritten as

$$
\chi=\frac{1}{3} \frac{U_{\mathrm{c}}}{U-U_{\mathrm{c}}},
$$

where we used the relation $T=2 U-1$ in the high-energy, homogeneous region, $U>U_{\mathrm{c}}=3 / 4$. We note that this form (82) of susceptibility can be also obtained by computing the $N$-body partition function and by using the minimum free energy principle for a functional of the single-body distribution function [26].

\section{B. Power-law tails}

The second example is a family of distributions having power-law tails as

$$
f_{v}(p)=\frac{1}{2 \pi} \frac{A_{v}\left(p_{0}\right)}{1+\left|p / p_{0}\right|^{v}} .
$$

To be precise, this family is not smooth at $p=0$ except for even $v$ and, hence, is not suitable to consider analytic continuation to obtain the dispersion function $D_{x}(0)$. However, we apply the linear response theory to this family formally.

The parameter $p_{0}$ is determined by the kinetic energy $K=$ $\iint\left(p^{2} / 2\right) f_{v}(p) d q d p$ as

$$
p_{0}=\left[\frac{2 K \sin (3 \pi / \nu)}{\sin (\pi / \nu)}\right]^{1 / 2},
$$

and the normalization factor $A_{v}\left(p_{0}\right)$ is expressed by

$$
A_{v}\left(p_{0}\right)=\frac{v}{2 \pi p_{0}} \sin \left(\frac{\pi}{v}\right)
$$

for $v>2$ [27]. This family gives a second-order phase transition at the critical energy

$$
U_{\mathrm{c}}^{v}=\frac{1}{2}+\frac{1}{4} \frac{\sin (\pi / \nu)}{\sin (3 \pi / \nu)}
$$

for $v>2$. The dispersion function is computed as

$$
D_{\nu}(0)=1+\pi \int_{-\infty}^{\infty} \frac{f_{v}^{\prime}(p)}{p} d p=1-\frac{1}{2 p_{0}^{2}},
$$

and, hence, the susceptibility is

$$
\chi_{v}=C_{v} \frac{U_{\mathrm{c}}^{v}}{U-U_{\mathrm{c}}^{v}},
$$

where the factor $C_{v}$ is

$$
C_{\nu}=\frac{\sin (\pi / \nu)}{\sin (\pi / \nu)+2 \sin (3 \pi / \nu)} .
$$

We remark that the critical energy (86) and the constant (89) coincide with ones for thermal equilibrium by taking $v=4$, which gives $\left(U_{\mathrm{c}}^{4}, C_{4}\right)=(3 / 4,1 / 3)$. Taking the limit of $v \rightarrow \infty$, 
the distribution (83) becomes a homogeneous waterbag and gives $\left(U_{\mathrm{c}}^{v}, C_{v}\right) \rightarrow(7 / 12,1 / 7)$. We also remark that $C_{v}$ is an decreasing function of $v$ and $v$ must be $v>3$ to converge energy $U$. The range of $C_{v}$ is, thus, $1 / 7<C_{v}<1$. In particular, in the interval $3<v<4$, where the critical energy $U_{\mathrm{c}}^{v}$ is larger than $3 / 4, C_{v}$ is larger than the value $1 / 3$ for thermal equilibrium.

\section{Lynden-Bell distribution}

The third example is a family of Lynden-Bell distributions

$$
f_{\mathrm{LB}}(p)=\frac{\eta_{0}}{e^{\alpha+\beta p^{2} / 2}+1} .
$$

This family is expected as a quasistationary state starting from a rectangle waterbag initial state $[16,17]$, and we parametrize this family by energy $U$ and the magnetization $M_{\mathrm{wb}}$ of the waterbag state. Distribution of the waterbag is written in the form

$$
f_{\mathrm{wb}}(q, p)=\left\{\begin{array}{l}
\eta_{0}\left(|q|<q_{0} \text { and }|p|<p_{0}\right), \\
0 \quad \text { (otherwise) }
\end{array}\right.
$$

The two parameters $q_{0}$ and $p_{0}$ determine the factors $\eta_{0}$, the magnetization $M_{\mathrm{wb}}$ and energy $U$ as

$$
\eta_{0}=\frac{1}{4 q_{0} p_{0}}, \quad M_{\mathrm{wb}}=\frac{\sin q_{0}}{q_{0}}, \quad U=\frac{p_{0}^{2}}{6}+\frac{1-M_{\mathrm{wb}}^{2}}{2} .
$$

The Lagrange multipliers $\alpha$ and $\beta$ included in (90) are determined by the two constraints

$$
\iint_{\mu} f_{\mathrm{LB}}(p) d q d p=1
$$

and

$$
\iint_{\mu} \frac{p^{2}}{2} f_{\mathrm{LB}}(p) d q d p+\frac{1}{2}=U
$$

We stress that the parameters $M_{\mathrm{wb}}$ and $U$ are used just to parametrize the family (90) for convenience of comparison with previous works, and the stationary state $f_{0}$ is set as $f_{\mathrm{LB}}$ instead of $f_{\mathrm{wb}}$. In other words, we do not consider the violent relaxation process from the waterbag to the Lynden-Bell distribution.

The family (90) has a tricritical point at $\left(M_{\mathrm{wb}}^{\mathrm{tc}}, U_{\mathrm{tc}}\right)$ on the parameter plane $\left(M_{\mathrm{wb}}, U\right)$ [28], and there is a second-order phase transition along an iso- $M_{\mathrm{wb}}$ line for $M_{\mathrm{wb}}>M_{\mathrm{wb}}^{\mathrm{tc}}$, and a first-order phase transition for $M_{\mathrm{wb}}<M_{\mathrm{wb}}^{\mathrm{tc}}$. Even for $M_{\mathrm{wb}}<$ $M_{\mathrm{wb}}^{\mathrm{tc}}$, a homogeneous Lynden-Bell distribution is locally stable in a high-energy region. The stable region is expressed by the inequality

$$
D_{x}(0)>0,
$$

and $D_{x}(0)=0$ gives the critical energy $U_{\mathrm{c}}\left(M_{\mathrm{wb}}\right)$ as shown in Fig. 1. Using this critical energy, the susceptibility $\chi$ is expressed by

$$
\chi=\frac{1-D_{x}(0)}{D_{x}(0)}=C\left(M_{\mathrm{wb}}\right) u^{-\gamma\left(M_{\mathrm{wb}}\right)}, \quad u=\frac{U-U_{\mathrm{c}}}{U_{\mathrm{c}}} .
$$

We check if the Curie-Weiss-like law, $\gamma\left(M_{\mathrm{wb}}\right)=1$, is satisfied even in QSSs by computing $D_{x}(0)$ and by direct temporal evolutions of the Vlasov equation.

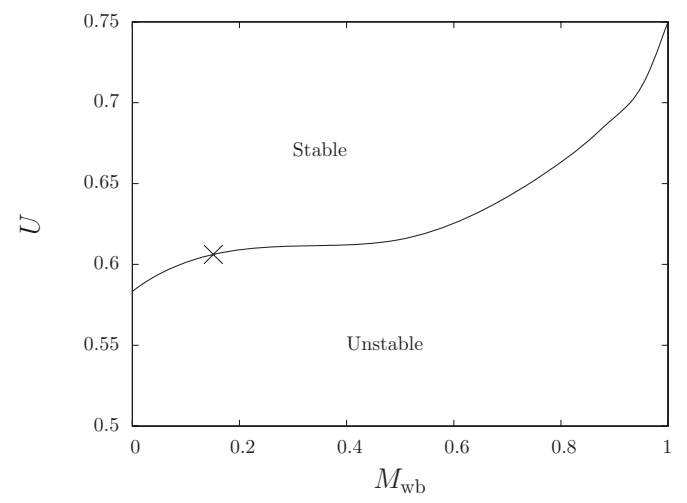

FIG. 1. Critical line determined by $D_{x}(0)=0$. The cross point represents the tricritical point [28]. The homogeneous state is stable or metastable in the upper side of the critical line and is unstable in the lower side.

The Vlasov equation is evolved by use of the semiLagrangian method [29]. We denote the number of grid points for $q$ and $p$ directions by $N_{q}$ and $N_{p}$, respectively. The time slice is fixed as $\Delta t=0.05$. We introduce cutoff for the $p$ direction and the computed interval of $p$ is $[-20,20]$. Strength of the external field is $h=0.005$.

The normalized response $M_{x} / h$, where $M_{x}=M_{1, x}$ in homogeneous case, is shown in Fig. 2 as a function of $u=\left(U-U_{\mathrm{c}}\right) / U_{\mathrm{c}}$ along the iso- $M_{\mathrm{wb}}$ line with $M_{\mathrm{wb}}=0.5$. The theoretical line seems almost straight, while semi-Lagrangian method gives nonstraight curve for small $u$, namely $u<0.1$. The present theory is valid for $\left|M_{x} / h\right| \lesssim 1$, and, hence, the small $u$ region is out of scope of the theory.

In order to compute the exponent $\gamma\left(M_{\mathrm{wb}}\right)$ and the factor $C\left(M_{\mathrm{wb}}\right)$, we apply the least-squares method for various intervals of $u$. Computed $\gamma\left(M_{\mathrm{wb}}\right)$ and $C\left(M_{\mathrm{wb}}\right)$ are exhibited in Fig. 3. The theoretical exponent $\gamma\left(M_{\mathrm{wb}}\right)$ is not the unity, but damps to the unity as the interval excludes low-energy region. We remark that, for the interval $u \in[1,10], \gamma\left(M_{\mathrm{wb}}\right)$

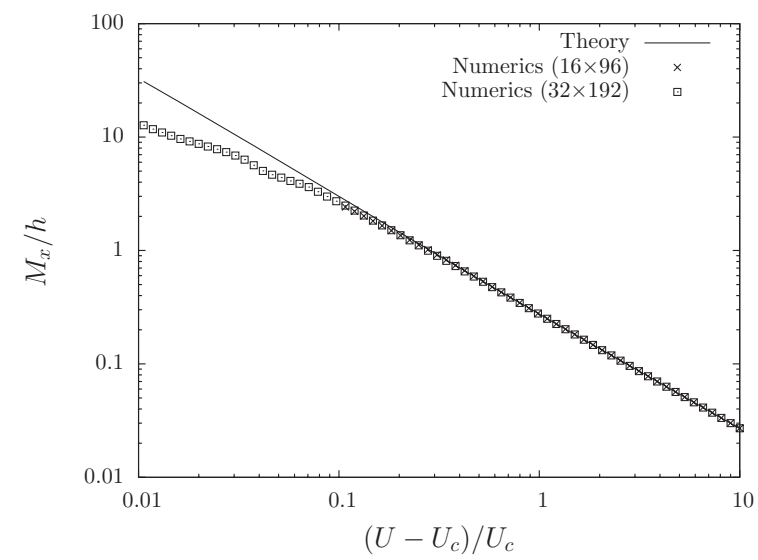

FIG. 2. Normalized response $M_{x} / h$ as a function of normalized energy $u=\left(U-U_{\mathrm{c}}\right) / U_{\mathrm{c}}$ along iso- $M_{\mathrm{wb}}$ line with $M_{\mathrm{wb}}=0.5, h=$ 0.005 . The line is obtained by use of the theory, and the points are obtained by use of the semi-Lagrangian method. Crosses are for $N_{q}=16$ and $N_{p}=96$, and squares for $N_{q}=32$ and $N_{p}=192$. The points are averages of $M_{x}(t)$ in the time interval $t \in[200,500]$. 

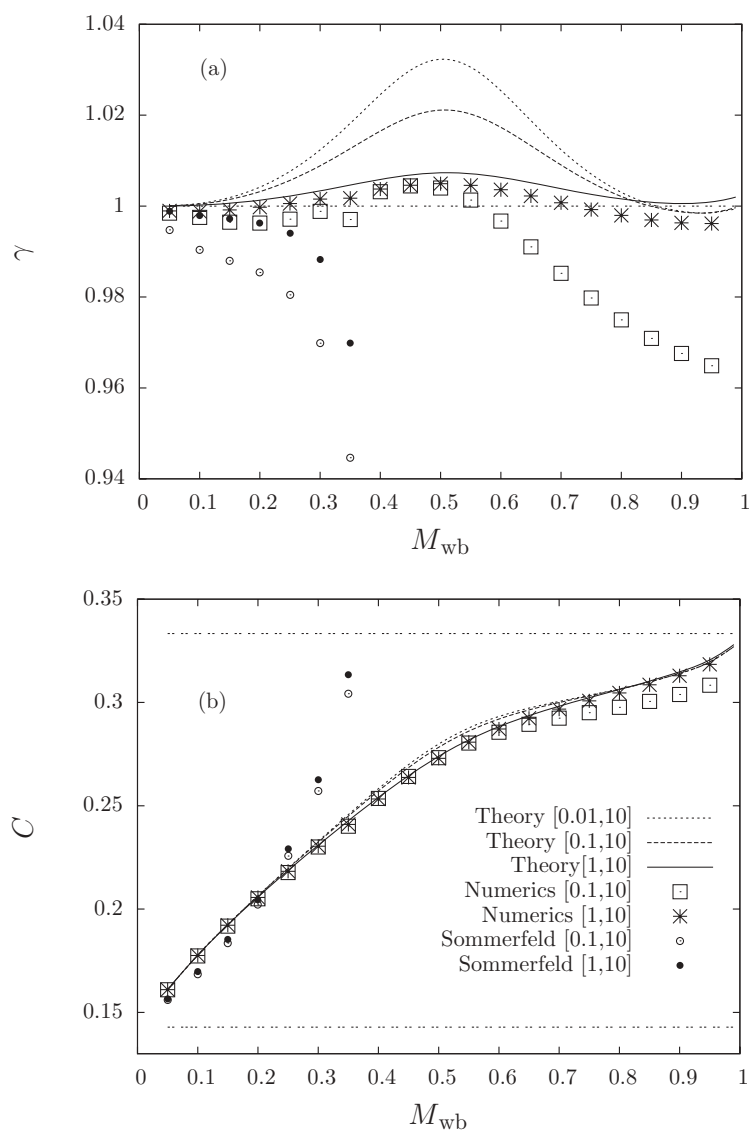

FIG. 3. $M_{\mathrm{wb}}$ dependence of (a) exponent $\gamma\left(M_{\mathrm{wb}}\right)$ and (b) factor $C\left(M_{\mathrm{wb}}\right)$, which are defined by (96). Lines are obtained by the theory, big points by the semi-Lagrangian method, and small circle points by Sommerfeld expansion reported in Ref. [21]. The dashed line is computed by use of the least-squares method applied to the interval $u \in[0.01,10]$. The broken line, square points, and white small points are for $u \in[0.1,10]$. Star points and black small points are for $u \in$ $[1,10]$. Two horizontal lines in the panel (b) represent $1 / 3$ (upper) and $1 / 7$ (lower), which are theoretically obtained factors for thermal equilibrium and waterbag, respectively.

and $C\left(M_{\mathrm{wb}}\right)$ for $N_{q}=16$ and $N_{p}=96$ coincide with ones for $N_{q}=32$ and $N_{p}=192$, respectively, though the result for the former is not reported. We therefore may conclude that a Curie-Weiss-like law, $M_{x} / h \propto 1 / u$, appears in an asymptotic high-energy region.

The factor $C\left(M_{\mathrm{wb}}\right)$ monotonically increases as $M_{\mathrm{wb}}$ increases and almost is not affected by the choice of the interval of $u$. In particular, in a high-energy region $u \in[1,10]$, the semi-Lagrangian method is in good agreement with the theory. The susceptibility, hence, is written in the Curie-Weiss-like law $\chi=C\left(M_{\mathrm{wb}}\right) / u$ in the high-energy region.

We also computed the exponent $\gamma\left(M_{\mathrm{wb}}\right)$ and the factor $C\left(M_{\mathrm{wb}}\right)$ from equations derived by the Sommerfeld expansion reported in Ref. [21]. The Sommerfeld expansion breaks around $M_{\mathrm{wb}} \simeq 0.2$ since a large $M_{\mathrm{wb}}$ gives a small $\beta$ in (90), while the expansion assumes a large $\beta$.

\section{Momentum deviation and $C$ factor}

We considered three types of distributions, thermal equilibrium, power-law tails and Lynden-Bell distributions, and the

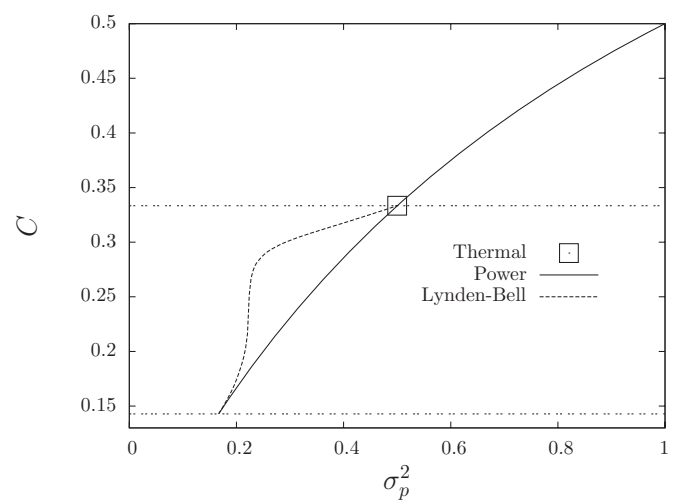

FIG. 4. Factor $C$ as a function of momentum deviation at the critical point. The square point is for thermal equilibrium, the solid line is for power tails, and the broken line is for Lynden-Bell.

Curie-Weiss-like law is satisfied, at least in the high-energy region. Factor $C$ depends on a considering family of stationary distributions, and it tends to increase as momentum deviation becomes large. Is there universality in the relation between factor $C$ and momentum deviation?

Factor $C$ is reported in Fig. 4 as a function of momentum deviation $\sigma_{p}^{2}$ at the critical point. It is true that $C$ is an increasing function of $\sigma_{p}^{2}$, but no universality is found. This nonuniversality suggests the possibility that a type of distribution family of QSSs could be detected by computing factor $C$ if the family has a second-order phase transition. However, we need further investigations of critical phenomena in QSSs to perform this detection.

\section{RESPONSE IN INHOMOGENEOUS CASE}

We now investigate the linear response in the inhomogeneous case. We use thermal equilibrium as a family of QSSs, which are expressed by

$$
f_{0}(q, p)=A e^{-\beta\left(p^{2} / 2-M_{0} \cos q\right)}
$$

where $A$ is the normalization factor. The 0 -th order magnetization $M_{0}$ points to the $x$ direction and must satisfy the self-consistent equation

$$
M_{0}=\iint f_{0}(q, p) \cos q d q d p .
$$

We consider the linear response to nonoscillating external fields in Sec. VIA, and resonance absorption by oscillating fields in Sec. VIB.

\section{A. Response to nonoscillating external field}

In the inhomogeneous case, the rotational symmetry breaks and we separately consider two types of external fields which point to the $x$ direction and to the $y$ direction.

An external field to the $x$ direction is expressed by $\left(h_{x}(t), h_{y}(t)\right)=(h \Theta(t), 0)$. The response $M_{1, x}$ is expressed by (74), and the concrete form of the dispersion function at $\omega=0, D_{x}(0)$, is written by using elliptic integrals as follows.

Let us introduce a new variable,

$$
k=\sqrt{\left(M_{0}+\epsilon\right) / 2 M_{0}},
$$




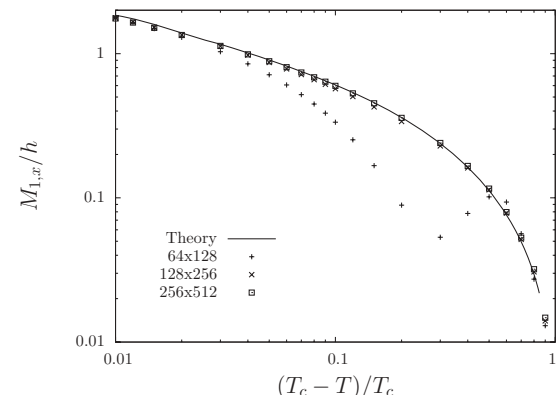

FIG. 5. Response $M_{1, x} / h$ as a function of $\left(T_{\mathrm{c}}-T\right) / T_{\mathrm{c}}$ with $T_{\mathrm{c}}=$ $1 / 2$. $h=0.005$. The line is obtained by the theory, and the points by the semi-Lagrangian method. The value of $M_{1, x}$ in the semiLagrangian method is averaged over the time interval $t \in[200,500]$. The numbers in panel represent the size of grid $N_{q} \times N_{p}$.

with the single-body energy

$$
\epsilon=\frac{p^{2}}{2}-M_{0} \cos q
$$

As we assumed before, the stationary state $f_{0}(q, p)$ depends on $q$ and $p$ through the single-body energy and, hence, through $k$ only, that is,

$$
f_{0}(q, p)=\tilde{f_{0}}(k) \text {. }
$$

Using $\tilde{f}_{0}$, the dispersion function $D_{x}(0)$ is written in the form

$$
\begin{aligned}
D_{x}(0)= & +\iint_{\mu} \frac{1}{p} \frac{\partial f_{0}}{\partial p}(q, p) \cos ^{2} q d q d p \\
& -\frac{4}{\sqrt{M_{0}}} \int_{1}^{\infty} \frac{K(1 / k)}{k}\left[\frac{2 k^{2} E(1 / k)}{K(1 / k)}+1-2 k^{2}\right]^{2} \\
& \times \frac{d \tilde{f}_{0}}{d k}(k) d k-\frac{4}{\sqrt{M_{0}}} \int_{0}^{1} K(k) \\
& \times\left[\frac{2 E(k)}{K(k)}-1\right]^{2} \frac{d \tilde{f}_{0}}{d k}(k) d k
\end{aligned}
$$

where $K(k)$ and $E(k)$ are the complete elliptic integrals of the first and the second kinds, respectively. The theoretical linear response is obtained by computing $D_{x}(0)$ and substituting it into Eq. (74). The theoretical prediction is reported in Fig. 5 with numerical results of the semi-Lagrangian method. The theoretical curve is in good agreement with numerics of the grid sizes $N_{q} \times N_{p}=128 \times 256$ and $256 \times 512$.

We also consider response to the external field to the $y$ direction, $\left(h_{x}(t), h_{y}(t)\right)=(0, h \Theta(t))$. The concrete form of $D_{y}(0)$ is

$$
D_{y}(0)=1+\iint_{\mu} \frac{1}{p} \frac{\partial f_{0}}{\partial p}(q, p) \sin ^{2} q d q d p,
$$

and $D_{y}(0)=0$ as shown in Ref. [30]. The susceptibility for the $y$ direction therefore diverges. This divergence is consistent with the fact that the magnetization vector $\left(M_{x}, M_{y}\right)$, which is initially set as $\left(M_{0}, 0\right)$, changes the direction and $M_{y}$ becomes finite by an external field $(0, h)$, even $h$ is arbitrary small.

\section{B. Resonance absorption}

We apply the linear response theory for extracting nonequilibrium dynamics in the unforced system through resonance absorption for oscillating external fields. The HMF system has "Landau dampings" even in inhomogeneous case, and there are several Landau poles corresponding to roots of the dispersion function, $\operatorname{det}(I-F(\omega))$. The imaginary part of the roots is negative if the stationary state $f_{0}$ is stable. The most important root is the one whose imaginary part is the largest, since the damping rate corresponding to the root is the smallest. We call this root the main root and denote $\omega_{L}=\omega_{1}+i \omega_{2}, \omega_{1}, \omega_{2} \in \mathbb{R}$. Detecting the main root has been explicitly done for inhomogeneous thermal equilibrium of the HMF model [24], but this detection is a hard task since logarithmic singularities appear in $F$ and $G$ functions. We therefore try to capture "Landau dampings" in inhomogeneous states from the viewpoint of resonance absorption.

Let us consider the external field vector pointing to the $x$ direction, that is, $\left(h_{x}(t), 0\right)$ and $h_{x}(t)$ is represented by $(70)$. In a short time interval, the external field $h_{x}(t)$ induces a small modification of magnetization denoted by $d M_{1, x}(t)$. The work by the external field is, hence, expressed by

$$
d W=h_{x}(t) d M_{1, x}(t),
$$

and the average over one period is computed by use of

$$
\begin{aligned}
\bar{W} & =\frac{\omega_{0}}{2 \pi} \int_{0}^{2 \pi / \omega_{0}} h_{x}(t) \frac{d M_{x}}{d t}(t) d t \\
& =\frac{-i h^{2} \omega_{0}}{4}\left[\frac{1-D_{x}\left(\omega_{0}\right)}{D_{x}\left(\omega_{0}\right)}-\frac{1-D_{x}\left(-\omega_{0}\right)}{D_{x}\left(-\omega_{0}\right)}\right],
\end{aligned}
$$

where we used asymptotic expression of $M_{1, x}(t),(73)$.

The dispersion function $D_{x}(\omega)$ satisfies the relation

$$
D_{x}\left(-\omega^{*}\right)=D_{x}(\omega)^{*},
$$

where $\omega^{*}$ is the complex conjugate of $\omega$. The work is, for a real $\omega_{0}$, therefore expressed by

$$
\bar{W}=\frac{h^{2} \omega_{0}}{2} \operatorname{Im}\left[\frac{1-D_{x}\left(\omega_{0}\right)}{D_{x}\left(\omega_{0}\right)}\right] .
$$

Moreover, if $\omega_{L}$ is a main root, then $-\omega_{L}^{*}=-\omega_{1}+i \omega_{2}$ is also a main root. Hence, we can write $D_{x}(\omega)$ in the form

$$
D_{x}(\omega)=\left(\omega-\omega_{L}\right)\left(\omega+\omega_{L}^{*}\right) \varphi(\omega) .
$$

To estimate the maximum work as a function of $\omega_{0}$, we introduce two assumptions: (i) $1-D_{x}\left(\omega_{0}\right) \simeq 1$ and (ii) $\varphi\left(\omega_{0}\right)$ is a constant $\varphi_{0}$. These assumptions lead us to the fact that $\varphi_{0}$ is a real number, and, consequently, $\bar{W}$ takes the maximum value

$$
\bar{W}_{\max }=\frac{h^{2}}{4 \omega_{2} \varphi_{0}}
$$

at frequency

$$
\omega_{0}= \pm\left|\omega_{L}\right|
$$

Numerical tests are performed by adding the oscillating external field of $H_{1}=-h \Theta(t) \cos \left(\omega_{0} t\right) \cos q$ with $h=0.01$. We expect that the system gets larger energy from the external field as frequency $\omega_{0}$ becomes closer to $\pm\left|\omega_{L}\right|$. Energy gain 


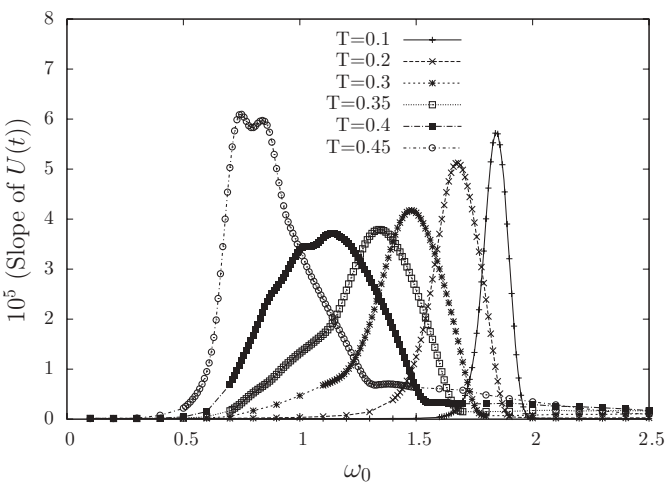

FIG. 6. Slope of $U(t)$ as a function of $\omega_{0}$. The slope is computed by the least-squares method in the time interval $t \in[0,100]$. A semiLagrangian method is performed with $N_{q}=128, N_{p}=256, \Delta t=$ 0.05 , and $h=0.01$.

over time is linear in the theory, and we compute the slope of $U(t)$ in $t \in[0,100]$ by use of the least-squares method. We selected a short time region to estimate the slope since large energy gain may modify the stationary state $f_{0}$.

The slopes are reported as functions of $\omega_{0}$ for several values of temperature $T$ in Fig. 6. We capture three qualitative features from the slopes: (i) The peak position shifts from right to left as $T$ increases. (ii) The graph for $T=0.45$ has two peaks and a sign of the double peaks can be observed in the graph for $T=0.4$. (iii) Height of the peak shows the $\mathrm{V}$ letter shape as a function of $T$. Considering the fact that $\left|\omega_{2}\right|$ is much smaller than $\left|\omega_{1}\right|$, and $\left|\omega_{L}\right|$ is close to $\left|\omega_{1}\right|$, the feature (i) directly reflects $T$ dependence of $\omega_{1}$. Similarly, (ii) captures discontinuous change of frequency of the main root around $T \simeq 0.38$, at which two pairs of main roots have the same imaginary part but a real part that differs. See Fig. 11 of Ref. [24] and Table I for (i) and (ii). The third feature (iii) may be explained by the maximum work of the external field, which is expressed by (109), and the turn in the V letter shape of $-\omega_{2}$ as a function of $T$. See Fig. 10 of Ref. [24] and Table I.

However, the peak position is quantitatively not in agreement with $\left|\omega_{L}\right|$. We introduced two assumptions in the way of deriving the frequency (110) that gives the maximum work, and they may cause this discrepancy. We remark that this discrepancy, that is the peak positions are slightly larger than $\left|\omega_{L}\right|$ for $T=0.4$ and 0.45 , is also observed in comparison between the Landau pole and direct numerical computation of frequency in Landau damping by $N$-body simulations [24].

TABLE I. Comparison between $\left|\omega_{L}\right|$ and peak positions of Fig. 6. Real and imaginary parts of $\omega_{L}$ are read from Figs. 11 and 10 of Ref. [24], respectively. For "Peak position in Fig. 6," two values, 0.75 and 0.84 , correspond to two peaks for $T=0.45$.

\begin{tabular}{clllll}
\hline \hline$T$ & 0.2 & 0.3 & 0.35 & 0.4 & 0.45 \\
\hline$\left|\omega_{1}\right|$ & 1.7 & 1.4 & 1.2 & 0.8 & 0.6 \\
$-\omega_{2}$ & 0.08 & 0.15 & 0.22 & 0.30 & 0.18 \\
$\left|\omega_{L}\right|$ & 1.7 & 1.4 & 1.2 & 0.9 & 0.6 \\
Peak position in Fig. 6 & 1.68 & 1.48 & 1.34 & 1.14 & $0.75 / 0.84$ \\
\hline \hline
\end{tabular}

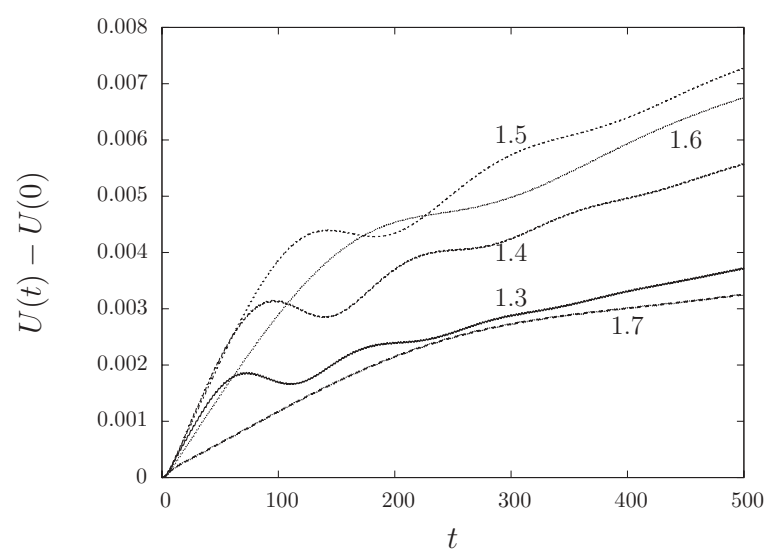

FIG. 7. Temporal evolution of energy, $U(t)-U(0)$, for some values of $\omega_{0}, T=0.3$. The numbers assigned to curves represent $\omega_{0}$. A semi-Lagrangian method is performed with $N_{q}=128, N_{p}=256$, $\Delta t=0.05$, and $h=0.01$.

We also remark that the energy gain is not completely linear in numerics as shown in Fig. 7, and further investigations are necessary to extract nonequilibrium dynamics precisely.

\section{SUMMARY AND DISCUSSIONS}

We proposed the linear response theory based on the Vlasov equation for spatially periodic one-dimensional Hamiltonian systems. The theory was examined for homogeneous and for inhomogeneous cases in the HMF model by comparing with direct numerical computations of the Vlasov equation.

As examples of homogeneous distributions, we considered three families of distributions: thermal equilibrium, power-law tails, and Lynden-Bell distributions. These families have stable or metastable homogeneous states, and such (meta-)stable states become unstable continuously by changing parameters. $\mathrm{We}$, hence, expected that the present linear response theory leads a Curie-Weiss-like law for the considering families. Indeed, the theory gives the exactly same susceptibility with equilibrium statistical mechanics for thermal equilibrium. The theory also predicts Curie-Weiss-like laws even in QSSs of power-law tails and of Lynden-Bell distributions. For the Lynden-Bell distributions, the theory and direct numerical simulations imply that the Curie-Weiss-like law holds in an asymptotically high-energy region. The coefficient of the Curie-Weiss-like laws tends to be large as momentum deviation of considering homogeneous stationary state becomes large. However, no universality is found in the coefficient as a function of the momentum deviation among the three tested families except for two special points of thermal equilibrium and homogeneous waterbag distribution. We note that the linear response theory is not guaranteed for large response and cannot discuss on divergence of the susceptibility at the critical point.

We emphasize that the linear response theory also gives good predictions for the inhomogeneous stationary state. Moreover, the resonance absorption in inhomogeneous thermal equilibrium is useful to investigate nonequilibrium dynamics of an unforced system. Energy gain by oscillating 
external fields with frequency $\omega_{0}$ has a peak around $\omega_{0}=\left|\omega_{L}\right|$, where $\omega_{L}$ is the main Landau pole, although estimation of the peak position is not complete quantitatively. However, in thermal equilibrium, temperature dependence of $\omega_{L}$ is qualitatively captured by this resonance absorption. Detecting the main Landau pole by use of the resonance absorption may have an advantage against direct computation of roots of dispersion relation, since the latter is a hard task in the inhomogeneous case in particular.

We neglected the case that the frequency $\omega_{0}$ of external field coincides with one of branch points of logarithmic singularities in inhomogeneous case. The logarithmic singularities give algebraic damping of the linear response by the same mechanism shown in Ref. [25], though the external forces are exerted eternally. Nevertheless, according to our preliminary numerical test, the algebraic damping could not be observed. This damping may not be robust since (i) we cannot set $\omega_{0}$ exactly on one of the branch points and (ii) the branch points are determined by 0 -th order stationary state but may be shifted effectively due to energy gain by the resonance absorption. Detailed analysis of this phenomenon is left for future work.

\section{ACKNOWLEDGMENTS}

The authors thank Shamik Gupta and Stefano Ruffo for fruitful discussions and for letting us know about their similar project. We also thank Freddy Bouchet for useful comments. S.O. acknowledges support of the Kyoto University Global COE Program: Informatics Education and Research Center for Knowledge-Circulating Society. Y.Y.Y. acknowledges the support of a Grant-in-Aid for Scientific Research (C) 23560069 .

\section{APPENDIX A: DERIVATION OF (15)}

The linear response of $B$ is simply written by

$\langle B\rangle_{1}(t)=-\iint_{\mu} d q d p B(q, p) \int_{0}^{t} e^{(t-s) \mathcal{L}_{0}} A(q, p, s) d s$,

where

$$
A(q, p, t)=\left\{\mathcal{V}\left[f_{1}\right](q, p, t)+H_{1}(q, p, t), f_{0}(q, p)\right\} .
$$

We derive (15) from (A1).

Let $A_{0}(q, p)$ be a smooth function, and $A(q, p, t)$ be a solution to the equation

$$
\frac{\partial A}{\partial t}+\left\{\mathcal{H}_{0}\left[f_{0}\right], A\right\}=0
$$

with the initial condition $A(q, p, 0)=A_{0}(q, p)$. Using the linear operator $\mathcal{L}_{0}$, the solution $A(q, p, t)$ is expressed by

$$
\begin{aligned}
A(q, p, t) & =e^{t \mathcal{L}_{0}} A_{0}(q, p)=e^{(t-s) \mathcal{L}_{0}} e^{s \mathcal{L}_{0}} A_{0}(q, p) \\
& =e^{(t-s) \mathcal{L}_{0}} A(q, p, s) .
\end{aligned}
$$

We derive another expression of the solution $A(q, p, t)$ by using a solution of the canonical equation of motion.

Let $(q(t), p(t))=\phi_{0}^{t}(q, p)$ be a solution to the canonical equation of motion

$$
\dot{q}=\frac{\partial \mathcal{H}_{0}\left[f_{0}\right]}{\partial p}(q, p), \quad \dot{p}=-\frac{\partial \mathcal{H}_{0}\left[f_{0}\right]}{\partial q}(q, p)
$$

with the initial condition $(q(0), p(0))=(q, p)$. Equation (A3) implies that $A$ is constant on this solution and, hence,

$$
\begin{aligned}
A(q, p, t) & =A(q(-t), p(-t), 0) \\
& =A(q(-(t-s)), p(-(t-s)), s) \\
& =A\left(\phi_{0}^{-(t-s)}(q, p), s\right)
\end{aligned}
$$

holds.

From Eqs. (A4) and (A6), we have the relation

$$
e^{(t-s) \mathcal{L}_{0}} A(q, p, s)=A\left(\phi_{0}^{-(t-s)}(q, p), s\right) .
$$

The above equation rewrites the linear response (A1) into the form

$$
\langle B\rangle_{1}(t)=-\int_{0}^{t} d s \iint_{\mu} d q d p B(q, p) A\left(\phi_{0}^{-(t-s)}(q, p), s\right) .
$$

Performing the canonical transform $\left(q^{\prime}, p^{\prime}\right)=\phi_{0}^{-(t-s)}(q, p)$, and using the fact that $\phi_{0}^{-(t-s)}$ is canonical and $d q^{\prime} \wedge d p^{\prime}=$ $d q \wedge d p$ holds accordingly, we have

$$
\langle B\rangle_{1}(t)=-\int_{0}^{t} d s \iint_{\mu} d q^{\prime} d p^{\prime} B\left(\phi_{0}^{(t-s)}\left(q^{\prime}, p^{\prime}\right)\right) A\left(q^{\prime}, p^{\prime}, s\right) .
$$

This equation is nothing but (15), and the derivation is completed.

\section{APPENDIX B: POLE SINGULARITY OF $\widehat{h}(\omega)$}

Let $h(t)$ be a smooth function and satisfy

$$
\lim _{t \rightarrow \infty} h(t)=h_{\infty} \quad \text { and } \quad \int_{0}^{\infty}\left|h^{\prime}(t)\right| d t<\infty .
$$

We replaced the constant $h$ with $h_{\infty}$ to avoid confusion. We prove that the Laplace transform of $h(t)$ is described by

$$
\widehat{h}(\omega)=\frac{h_{\infty}}{-i \omega}+\varphi(\omega),
$$

where all the poles of $\varphi(\omega)$ are in the lower half $\omega$ plane.

To prove (B2), we show the relation

$$
\lim _{\omega \rightarrow 0}(-i \omega) \int_{0}^{\infty} e^{i \omega t} h(t) d t=h_{\infty} .
$$

The proof can be done by integrating by parts as follows:

$$
\begin{aligned}
-i \omega \int_{0}^{\infty} e^{i \omega t} h(t) d t & =\left[-e^{i \omega t} h(t)\right]_{t=0}^{\infty}+\int_{0}^{\infty} e^{i \omega t} h^{\prime}(t) d t \\
& =h(0)+\int_{0}^{\infty} e^{i \omega t} h^{\prime}(t) d t,
\end{aligned}
$$

where we used the condition $\operatorname{Im} \omega>0$, and $h^{\prime}(t)$ is the derivative of $h(t)$. Taking the limit $\omega \rightarrow 0$ and using the dominated convergence theorem, we have

$$
\lim _{\omega \rightarrow 0}(-i \omega) \int_{0}^{\infty} e^{i \omega t} h(t) d t=h(0)+\int_{0}^{\infty} h^{\prime}(t) d t,
$$

and this equation proves (B3).

To complete the proof of (B2), let us check other poles of $\widehat{h}(\omega)$. Suppose that $\omega_{p}$ is a pole. We consider three cases: (i) $\operatorname{Im} \omega_{p}>0$, (ii) $\operatorname{Im} \omega_{p}=0$, and (iii) $\operatorname{Im} \omega_{p}<0$. Performing the inverse Laplace transform, we can reject the cases (i) 
and (ii) except for $\omega_{p}=0$, since the existence of such a pole breaks the condition (B1). Consequently, (B2) has been proved.

Case (iii) is possible, but the contribution from this pole gives an exponentially decreasing term for $M_{1, x}(t)$. We then have shown that the asymptotic value of $M_{1, x}(t)$ is determined by the pole $\omega_{p}=0$ when the condition (B1) holds.

\section{APPENDIX C: SKETCH OF DERIVING (102) AND (103)}

Using the angle-action variables $(\theta, J), D_{x}(0)$ and $D_{y}(0)$ are written in the forms

$$
\begin{aligned}
& D_{x}(0)=1+2 \pi \sum_{m \in \mathbb{Z} \backslash\{0\}} \int_{L} \frac{f_{0}^{\prime}(J)}{\Omega(J)}\left|C^{m}(J)\right|^{2} d J, \\
& D_{y}(0)=1+2 \pi \sum_{m \in \mathbb{Z} \backslash\{0\}} \int_{L} \frac{f_{0}^{\prime}(J)}{\Omega(J)}\left|S^{m}(J)\right|^{2} d J,
\end{aligned}
$$

respectively. According to Appendix B of Ref. [24] and the periodicity of elliptic functions, the functions $\cos q(\theta, J)$ and $\sin q(\theta, J)$ are $2 \pi$ periodic with respect to $\theta$, and the relations

$$
\begin{aligned}
& 2 \pi \sum_{m \in \mathbb{Z} \backslash\{0\}}\left|C^{m}(J)\right|^{2}=\int_{-\pi}^{\pi} \cos ^{2} q(\theta, J) d \theta-2 \pi\left|C^{0}(J)\right|^{2}, \\
& 2 \pi \sum_{m \in \mathbb{Z} \backslash\{0\}}\left|S^{m}(J)\right|^{2}=\int_{-\pi}^{\pi} \sin ^{2} q(\theta, J) d \theta-2 \pi\left|S^{0}(J)\right|^{2},
\end{aligned}
$$

are obtained straightforwardly by using Parseval's theorem. Moreover, using explicit forms $\cos q(\theta, J)$ and $\sin q(\theta, J)$ exhibited in the paper [24], one can get

$$
\begin{aligned}
2 \pi & \int_{L} \frac{f_{0}^{\prime}(J)}{\Omega(J)}\left|C^{0}(J)\right|^{2} d J \\
= & \frac{1}{2 \pi} \int_{0}^{\infty} d k \frac{1}{\Omega(k)} \frac{d \tilde{f_{0}}}{d k}(k)\left(\int_{-\pi}^{\pi} \cos ^{2} q(\theta, J(k)) d \theta\right)^{2} \\
= & \frac{4}{\sqrt{M_{0}}} \int_{1}^{\infty} \frac{K(1 / k)}{k}\left(\frac{2 k^{2} E(1 / k)}{K(1 / k)}+1-2 k^{2}\right)^{2} \frac{d \tilde{f}_{0}}{d k}(k) d k \\
& +\frac{4}{\sqrt{M_{0}}} \int_{0}^{1} K(k)\left(\frac{2 E(k)}{K(k)}-1\right)^{2} \frac{d \tilde{f}_{0}}{d k}(k) d k,
\end{aligned}
$$

and

$$
2 \pi\left|S^{0}(J(k))\right|^{2}=\frac{1}{2 \pi}\left(\int_{-\pi}^{\pi} \sin q(\theta, J(k)) d \theta\right)^{2}=0,
$$

respectively. Substituting (C2), (C3), and (C4) into (C1), we obtain

$$
\begin{aligned}
D_{x}(0)= & +\int_{L} d J \frac{f_{0}^{\prime}(J)}{\Omega(J)} \int_{-\pi}^{\pi} \cos ^{2} q(\theta, J) d \theta-\frac{4}{\sqrt{M_{0}}} \\
& \times \int_{1}^{\infty} \frac{K(1 / k)}{k}\left[\frac{2 k^{2} E(1 / k)}{K(1 / k)}+1-2 k^{2}\right]^{2} \frac{d \tilde{f}_{0}}{d k}(k) d k \\
& -\frac{4}{\sqrt{M_{0}}} \int_{0}^{1} K(k)\left[\frac{2 E(k)}{K(k)}-1\right]^{2} \frac{d \tilde{f}_{0}}{d k}(k) d k
\end{aligned}
$$

and

$$
D_{y}(0)=1+\int_{L} d J \frac{f_{0}^{\prime}(J)}{\Omega(J)} \int_{-\pi}^{\pi} \sin ^{2} q(\theta, J) d \theta .
$$

We rewrite the terms in $D_{x}(0)$ and $D_{y}(0)$ which are

$$
\begin{aligned}
& \int_{L} d J \frac{f_{0}^{\prime}(J)}{\Omega(J)} \int_{-\pi}^{\pi} \cos ^{2} q(\theta, J) d \theta, \\
& \int_{L} d J \frac{f_{0}^{\prime}(J)}{\Omega(J)} \int_{-\pi}^{\pi} \sin ^{2} q(\theta, J) d \theta,
\end{aligned}
$$

respectively, by using the coordinate $(q, p)$ as follows. We keep in mind that a stable stationary solution $f_{0}$ to the Vlasov equation depends on the coordinate $(q, p)$ or on the action coordinate $J$ only through a single-body energy $\epsilon$. We, hence, write $\tilde{f}_{0}(\epsilon(J))=f_{0}(J)$ or $\tilde{f}_{0}(\epsilon(q, p))=f_{0}(q, p)$. Let us start from rewriting the term in $D_{x}(0)$ as

$$
\begin{array}{rl}
\int_{L} & d J \frac{f_{0}^{\prime}(J)}{\Omega(J)} \int_{-\pi}^{\pi} \cos ^{2} q(\theta, J) d \theta, \\
& =\int_{L} d J \int_{-\pi}^{\pi} d \theta \frac{d \tilde{f}_{0}}{d \epsilon}(\epsilon(J)) \cos ^{2} q(\theta, J), \\
& =\iint_{\mu} \frac{d \tilde{f}_{0}}{d \epsilon}(\epsilon(J)) \cos ^{2} q(\theta, J) d \theta d J, \\
& =\iint_{\mu} \frac{1}{p} \frac{\partial f_{0}}{\partial p}(q, p) \cos ^{2} q d q d p .
\end{array}
$$

In the way of computations we used the facts that $d \epsilon(J) / d J=$ $\Omega(J)$ and $d \theta \wedge d J=d q \wedge d p$. Additionally, we should note that $d \tilde{f}_{0} / d \epsilon$ is assumed to have no singularity for any $J \geqslant 0$, and, hence, the integral path $L$ lies on the real $J$ axis. A set $[-\pi, \pi) \times L$, therefore, coincides with the whole $\mu$ space. A similar computation gives

$$
\begin{aligned}
& \int_{L} d J \frac{f_{0}^{\prime}(J)}{\Omega(J)} \int_{-\pi}^{\pi} \sin ^{2} q(\theta, J) d \theta \\
& =\iint_{\mu} \frac{1}{p} \frac{\partial f_{0}}{\partial p}(q, p) \sin ^{2} q d q d p .
\end{aligned}
$$

We therefore obtain (102) and (103).
[1] A. Campa, T. Dauxois, and S. Ruffo, Phys. Rep. 480, 57 (2009).

[2] J. Binney and S. Tremaine, Galactic Dynamics, 2nd ed. (Princeton University Press, Princeton, NJ, 2008).

[3] T. Daxois, S. Ruffo, E. Arimond, and M. Wilkens, Dynamics and Thermodynamics of Systems with Long-Range Interactions, Lecture Notes in Physics (Springer, Berlin, 2002).
[4] Y. Y. Yamaguchi, J. Barré, F. Bouchet, T. Dauxois, and S. Ruffo, Physica A 337, 36 (2004),

[5] J. Barré, F. Bouchet, T. Dauxois, S. Ruffo, and Y. Y. Yamaguchi, Physica A 365, 177 (2006).

[6] A. Turchi, D. Fanelli, and X. Leoncini, Commun. Nonlinear Sci. Numer. Simulat. 16, 4718 (2011). 
[7] M. Kastner, Phys. Rev. Lett. 106, 130601 (2011).

[8] W. Braun and K. Hepp, Commun. Math. Phys. 56, 101 (1977).

[9] R. L. Dobrushin, Funct. Anal. Appl. 13, 115 (1979).

[10] H. Spohn, Large Scale Dynamics of Interacting Particles (Springer-Verlag, Berlin, 1991).

[11] D. Lynden-Bell, Mon. Not. R. Astron. Soc. 136, 101 (1967).

[12] Y. Levin, R. Pakter, and F. B. Rizzato, Phys. Rev. E 78, 021130 (2008).

[13] Y. Y. Yamaguchi, Phys. Rev. E 78, 041114 (2008).

[14] M. Joyce and T. Worrakitpoonpon, Phys. Rev. E 84, 011139 (2011).

[15] P. H. Chavanis, Eur. Phys. J. B 53, 487 (2006).

[16] A. Antoniazzi, D. Fanelli, J. Barré, P. H. Chavanis, T. Dauxois, and S. Ruffo, Phys. Rev. E 75, 011112 (2007).

[17] A. Antoniazzi, D. Fanelli, S. Ruffo, and Y. Y. Yamaguchi, Phys. Rev. Lett. 99, 040601 (2007).

[18] F. Staniscia, P. H. Chavanis, and G. De Ninno, Phys. Rev. E 83, 051111 (2011).

[19] R. Kubo, J. Phys. Soc. Jpn. 12, 570 (1957).
[20] R. Kubo, M. Toda, and N. Hashitsume, Statistical Physics II: Nonequilibrium Statistical Mechanics (Springer, Berlin, 1985).

[21] A. Patelli, S. Gupta, C. Nardini, and S. Ruffo, Phys. Rev. E 85, 021133 (2012).

[22] L. D. Landau, J. Phys. U.S.S.R. 10, 25 (1946); Collected papers of L. D. Landau, edited and with an introduction by D. Ter Haar (Pergamon Press, Oxford, 1965).

[23] L. P. Pitaevski and E. M. Lifshitz, Physical Kinetics (Butterworth-Heinemann, Oxford, 1981).

[24] J. Barré, A. Olivetti, and Y. Y. Yamaguchi, J. Stat. Mech. (2010) $\mathrm{P} 08002$.

[25] J. Barré, A. Olivetti, and Y. Y. Yamaguchi, J. Phys. A: Math. Gen. 44, 405502 (2011).

[26] P. H. Chavanis, Eur. Phys. J. B 80, 275 (2011).

[27] Y. Y. Yamaguchi, F. Bouchet, and T. Dauxois, J. Stat. Mech. (2007) P01020.

[28] S. Ogawa and Y. Y. Yamaguchi, Phys. Rev. E 84, 061140 (2011).

[29] P. de Buyl, Commun. Nonlinear Sci. Numer. Simulat. 15, 2133 (2010).

[30] A. Campa and P. H. Chavanis, J. Stat. Mech. (2010) P06001. 